\title{
Nanometer focusing properties of Fresnel zone plates described by dynamical diffraction theory
}

\author{
F. Pfeiffer and C. David \\ Paul Scherrer Institut, CH-5232 Villigen, Switzerland \\ J. F. van der Veen \\ Paul Scherrer Institut, CH-5232 Villigen, and ETH-Zürich, Switzerland \\ C. Bergemann \\ Cavendish Laboratory, University of Cambridge, J. J. Thomson Avenue, Cambridge CB3 OHE, United Kingdom
}

(Received 10 February 2006; published 26 June 2006)

\begin{abstract}
The x-ray focusing properties of linear Fresnel zone plates have been derived by solving the Helmholtz equation for the field propagating through the zones. We consider the imaging of a point object into the first diffraction order of a volume zone plate having its zones parallel to the optical axis. For plane wave illumination, the focal spot size is limited by the same material-dependent but wavelength-independent value that affects waveguide focusing. In marked contrast, for the one-to-one imaging condition, corresponding to specular reflection of the $\mathrm{x}$ rays from the zone boundaries, the image is found to have a minimal spot size approximately equal to the outermost zone width. Unlike x-ray waveguides, zone plates therefore do not appear to possess a fundamental limit to the smallest spot size to which they can focus.
\end{abstract}

DOI: 10.1103/PhysRevB.73.245331

PACS number(s): 41.50.+h, 07.85.Qe, 61.10.-i

\section{INTRODUCTION}

At synchrotron radiation facilities worldwide, there are an increasing number of research activities requiring hard $\mathrm{x}$-ray beams of small spot size. While x-ray absorption, fluorescence, and diffraction measurements at a third-generation source can be typically performed using beams of micrometer size, for specific applications one has already achieved approximately $50 \mathrm{~nm}$ with refractive lenses, ${ }^{1,2} 30-100 \mathrm{~nm}$ with reflecting Kirkpatrick-Baez mirrors, ${ }^{3}$ typically 25-40 nm with waveguides, ${ }^{4,5}$ down to $100 \mathrm{~nm}$ with Fresnel zone plates (FZPs), ${ }^{6,7}$ and $30 \mathrm{~nm}$ with multilayer Laue lenses. ${ }^{8}$ For FZPs serving as an objective lens in a full-field soft x-ray microscope, Chao et al. have recently achieved a resolution below $15 \mathrm{~nm} .{ }^{9}$ The question arises: What is the theoretical resolution limit for each of these devices? Ideally one would like to resolve down to, e.g., $1 \mathrm{~nm}$, and one has even discussed the possibility of focusing the extremely brilliant beam of a future fourth-generation source (an energyrecoverable LINAC or an $\mathrm{x}$-ray free electron laser) to a spot of just a few atoms wide. ${ }^{10}$ This would make x-ray microscopy, either in the full-field or the scanning mode, a true competitor of high-resolution electron microscopy, with the advantages of a larger depth of field and a greater element specificity.

Previously we have analyzed the focusing properties of tapered $\mathrm{x}$-ray waveguides, and we concluded that there is a fundamental limit of 7-10 nm to the smallest achievable spot size, its exact value depending on the electron density and the geometry of the confining material. ${ }^{11}$ The focusing limit arises from a smearing of the confined wave function for very small waveguide apertures over a length scale of $W_{c}=\lambda / 2 \theta_{c}$, with $\theta_{c}$ the critical angle for total reflection from the confining walls. Here, $\theta_{c}$ has the physical meaning of a numerical aperture (NA) of the focusing device. We conjectured that the same focusing limit also applies to other $\mathrm{x}$-ray optics such as refractive lenses and Fresnel zone plates
(FZPs). A recent theoretical analysis of refractive lenses, however, revealed that although this is true for a single lens, a series of such lenses, whose aperture is adiabatically matched to the converging beam size, can focus well below the theoretical limit for waveguides, corresponding to a much larger NA than is given by $\theta_{c}{ }^{12}$ As FZPs can be regarded as a set of coupled waveguides of varying width, they may exhibit a similar delocalization of the wave function in the outer zones, if the latter become as small as $\sim W_{c}$. To what extent this sets a limit to the focus size or the spatial resolution achievable with FZPs has not yet been investigated.

Here we report on the focusing properties of linear zone plates in the high-resolution limit by numerically solving the parabolic wave equation. ${ }^{13}$ We consider incident plane waves as well as cylindrical waves emerging from an on-axis line source oriented parallel to the FZP zones at finite distance, then propagate the waves through the volume zone plate, and finally propagate them through the free image space. Previous authors derived the focusing properties of volume-FZPs by use of a dynamical coupled-wave theory, assuming the zone width to be essentially constant over a small area of the FZP. ${ }^{14,15}$ The coupled wave theory predicts the amplitude and phase of the wavefield as a function of zone number, and one may reconstruct the image by applying the HuygensFresnel principle to the secondary waves emitted from the FZP exit. The coupled wave theory shows that the diffraction efficiency of the FZP in a given order generally is strongly dependent on the zone height and the local Bragg condition, i.e., on the local tilt angle of the zones with respect to the inand outgoing rays. Moreover, a decreasing local diffraction efficiency for the outer zones results in a restricted NA and therefore in a lower resolution (larger spot size). Coupled wave theory has been used for optimizing the diffraction efficiency of zone plates intended as objective lenses in fullfield x-ray microscopes. ${ }^{15,16}$ The adverse effects of zone roughness and fuzziness on the focusing size have been in- 


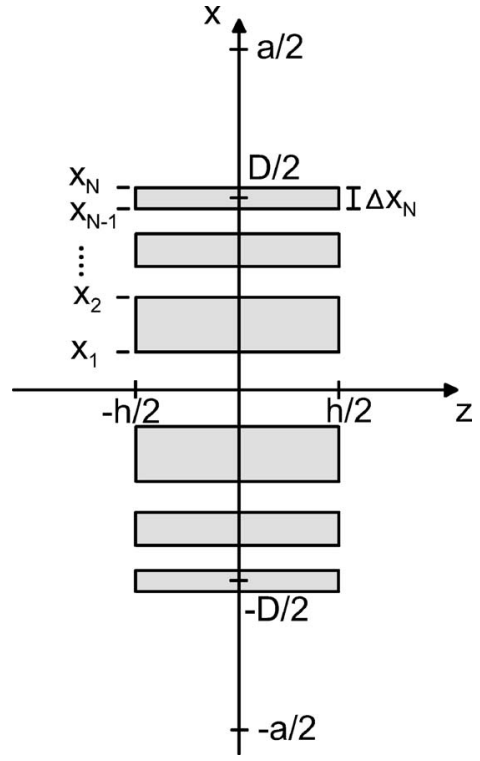

FIG. 1. Definition of the variables and the coordinate system.

vestigated by numerical solution of the wave equation. ${ }^{17}$

We present a method of propagating wave functions through linear FZPs which is based on finding the eigenstates and energy eigenvalues for the wave functions within the entire array of potential wells that form the zone plate. The assumption of a locally constant zone width as in coupled wave theory is not made, but for ease of computation the planes of the zones are assumed to be lying parallel to the optical axis. The focusing limits are explored for incident wavefronts emerging from on-axis line sources at various distances.

\section{THEORY OF THE PROPAGATION OF X RAYS THROUGH ZONE PLATES}

We consider a point source $P$ at some distance from a vertically linear zone plate, emitting radiation that is linearly polarized along the horizontal $y$ axis. As stated above, the FZP has its zone boundaries oriented parallel to the optical axis along the $z$ direction. The $z=0$ plane goes through the center of the FZP, which is assumed to be of thickness $h$. There are in total $N$ zones, the outer one being of width $\Delta x_{N}$. For the nomenclature used we refer to Fig. 1.

The number of parameters that could be varied to find an optimum zone plate geometry in terms of resolution and diffraction efficiency is rather large. Besides the fact that we only consider linear FZPs, we make several additional assumptions to limit the parameter space: (i) We assume that the FZP is a pure phase object. We describe its rectangular zone profile by a position-dependent refractive index $n(x)$ $=1-\delta(x)$, where the refractive index decrement $\delta$ is constant over a single zone width. (ii) We take the height $h$ of the zones such that the waves, upon exiting the zone material, have undergone a phase difference of $\pi$, i.e., $h=\lambda /(2 \delta)$. This yields maximum diffraction efficiency in all diffraction orders (except for the zeroth order) of the FZP in the kinematic diffraction approximation of a "thin" zone plate. This is not generally the case. For instance in the limit of a "thick" FZP, the height for optimum zone thickness is larger. ${ }^{18}$ Taking this effect into account would make the optimum zone height dependent of its width, which would greatly complicate the calculations, without giving additional information about the possible existence of a fundamental resolution limit. (iii) We restrict our calculations to FZPs with a (local) duty cycle of 0.5 , i.e., half of the zone plates volume is filled with matter and half is empty [see Eq. (1)]. Again we accept that this may not be the optimum FZP geometry, for the sake of simplicity.

Let $f$ be the focal distance of the FZP for the first order in the kinematic diffraction approximation (optically thin FZP). In order to achieve a phase difference of $\pi$ between two consecutive zones, which corresponds with an optical path difference of $\lambda / 2$, the zone boundaries are positioned at

$$
x_{\nu}= \pm \frac{1}{k}\left[(2 \nu-1) \pi k f+\left(\nu-\frac{1}{2}\right)^{2} \pi^{2}\right]^{1 / 2}, \quad \nu=1,2,3, \ldots, N
$$

Here, $k=2 \pi / \lambda$ is the magnitude of the wave vector and $N$ is the total number of zones. The width $\Delta x_{\nu}$ of zone $\nu$ is given by $\Delta x_{\nu} \equiv x_{\nu}-x_{\nu-1}$. Below we present a simple imaging theory which predicts the wavefield at the image point. The purpose of our calculations is to determine the image spot size for different outermost zone widths $\Delta x_{N}$ and source distances $s$, with the aim to establish whether there are any fundamental limits. We note that some of the FZPs considered here are technically not yet feasible; the smallest outer zone width achieved so far for a full FZP is $15 \mathrm{~nm},{ }^{9}$ and aspect ratios $h / \Delta x_{N}$ higher than 10 are challenging, when lithographic techniques are used. Nonetheless, we believe that our considerations are useful for future design strategies. Especially the approach to use sectioned multilayers for the focusing of $\mathrm{x}$ rays ${ }^{18}$ is not subject to the limitations mentioned above.

Our approach is based on the parabolic approximation in the Helmholtz equation, enabling us to write the latter in the form of the Schrödinger equation. ${ }^{11}$ The problem then becomes equivalent to finding the quantum wave function for an array of potential wells. The Helmholtz equation is given by

$$
\nabla^{2} u+n^{2} k^{2} u=0,
$$

with $\nabla^{2}=\partial^{2} / \partial x^{2}+\partial^{2} / \partial z^{2}$ the Laplace operator, $n(x)=1-\delta(x)$ the refractive index (no absorption), and $u$ the scalar wavefield which represents the component $E_{y}$ of the electric field $\mathbf{E}=\left(0, E_{y}, 0\right)$ parallel to the zones. For the solution $u$ of Eq. (2) we make the ansatz

$$
u(x, z)=\Psi(x, z) e^{-\mathrm{i} k z},
$$

with $\Psi(x, z)$ the envelope wave function that slowly varies with $z$. Substitution into Eq. (2) and neglecting $\partial^{2} \Psi / \partial z^{2}$ results in a wave equation that has the form of Schrödinger's equation,

$$
H \Psi=-\frac{\mathrm{i}}{k} \frac{\partial \Psi}{\partial z},
$$

in which the Hamiltonian 


$$
H \equiv H_{0}+V(x) \equiv-\frac{1}{2 k^{2}} \frac{\partial^{2}}{\partial x^{2}}+\delta(x)
$$

consists of a free-space kinetic energy contribution $H_{0}$ and a potential term $V(x)$. In Eq. (4), $k$ and $z$ replace $1 / \hbar$ and time $t$, respectively, and the mass $m=1$.

To neglect the $\partial^{2} \Psi / \partial z^{2}$ term is known as the slowly varying envelope approximation; ${ }^{19}$ this is frequently used in the beam propagation method. The approximation is known to be accurate if the variations in the refractive index are small $(\Delta n / n \ll 1)$ and if the angular spectrum of the field is narrow $\left(k_{x} / k \ll 1\right)$. Both conditions are generally met in X-ray optics.

The $z$ independence of $n(x)$ inside the FZP enables the determination of the eigenstates of the Hamiltonian in Eq. (5) within the entire zone plate in a straightforward manner. By separation of variables,

$$
\Psi(x, z)=e^{\mathrm{i} k E z} \psi(x),
$$

we obtain the time-independent Schrödinger equation

$$
H \psi=E \psi \text {. }
$$

Let $\left\{\psi_{n}\right\}$ be the eigenfunctions of $H$ and $\left\{E_{n}\right\}$ the corresponding eigenvalues

$$
H \psi_{n}=E_{n} \psi_{n} .
$$

The incoming wavefield $\left\langle x \mid \Psi_{\text {in }}\right\rangle \equiv \Psi_{\mathrm{n}}(x, z=-h / 2)$ is decomposed in eigenfunctions

$$
\left|\Psi_{\text {in }}\right\rangle=\sum_{n}\left\langle\psi_{n} \mid \Psi_{\text {in }}\right\rangle\left|\psi_{n}\right\rangle
$$

After propagation within the zone plate over its thickness $h$ the wave function has evolved to the exit wavefield $\left\langle x \mid \Psi_{\mathrm{ex}}\right\rangle \equiv \Psi_{\mathrm{ex}}(x, z=h / 2)$, with

$$
\left|\Psi_{\mathrm{ex}}\right\rangle=\sum_{n}\left\langle\psi_{n} \mid \Psi_{\mathrm{in}}\right\rangle e^{\mathrm{i} k E_{n} h}\left|\psi_{n}\right\rangle
$$

Behind the FZP, the field propagates as a free wave. Let $\left\{\chi_{n}\right\}$ and $\left\{E_{n}^{0}\right\}$ be the eigenstates and the corresponding eigenvalues of the free-space Hamiltonian $H_{0}$, satisfying

$$
H_{0} \chi_{n}=E_{n}^{0} \chi_{n} .
$$

At a distance $z_{0}$ behind the FZP, the wave function has evolved into $\left\langle x \mid \Psi_{z_{0}}\right\rangle \equiv \Psi\left(x, z=z_{0}\right)$, with

$$
\left|\Psi_{z_{0}}\right\rangle=\sum_{m}\left\langle\chi_{m} \mid \Psi_{\mathrm{ex}}\right\rangle e^{\mathrm{i} k E_{m}^{0}(z-h / 2)}\left|\chi_{m}\right\rangle
$$

The image is found at the distance $z_{\max }$ at which $|\Psi(x=0, z)|^{2}$ has its maximum. The limiting value of the width, i.e., the resolution, is determined by the highest spatial frequency transmitted by the FZP.

As seen in the Appendix, the eigenstates $\left\{\chi_{n}\right\}$ outside the FZP are standing waves with wave vector $q_{n}=(2 n-1) \pi / a$, with $a$ being the width of the interval over which the eigenstates of $H_{0}$ are defined [Eqs. (A2) and (A4)]. It is therefore useful to view the exit wave in Fourier space and define the Fourier amplitude spectrum

$$
\left|\Psi_{\mathrm{ex}}\left(q_{n}\right)\right| \equiv\left|\left\langle\chi_{n} \mid \Psi_{z_{0}}\right\rangle\right|=\left|\left\langle\chi_{n} \mid \Psi_{\mathrm{ex}}\right\rangle\right| .
$$

The cutoff wave vector $q$ beyond which $\Psi_{\text {ex }}\left(q_{n}\right)$ falls off determines the resolution.

The energy diffracted into the first order equals

$$
P=\int_{-x_{w} / 2}^{+x_{w} / 2}\left|\Psi\left(x, z_{\max }\right)\right|^{2} \mathrm{~d} x,
$$

where the integration interval $\left[-x_{w} / 2,+x_{w} / 2\right]$ should be chosen to be large enough to include the majority of intensity diffracted into the first order without significant contributions of the zeroth, negative, or higher positive diffraction orders. ${ }^{20}$ Normalizing to the energy passing through the FZP width,

$$
P_{0}=\int_{-D / 2}^{+D / 2}\left|\Psi_{\text {in }}(x)\right|^{2} \mathrm{~d} x,
$$

we obtain the diffraction efficiency in the first order,

$$
\eta=\frac{P}{P_{0}} .
$$

This value is to be compared with $\eta=4 / \pi^{2}=0.405$ for kinematic diffraction into the first order from a square-wave, $\pi$-phase shifting profile in the absence of absorption. ${ }^{21}$ However, it should be noted that efficiencies approaching unity are possible in the dynamical limit for zone plates of optimal geometry, when the Bragg condition is fulfilled.

\section{NUMERICAL IMPLEMENTATION AND RESULTS}

Wavefields have been propagated through the FZPs listed in Table I for two imaging geometries (Fig. 2): (a) a plane incident wave from a line source at infinite distance $z \rightarrow-\infty$ and (b) a cylindrical wave emerging from a source located at the position $z=-2 f$. According to the lens formula, illumination by plane waves should yield an image at the focal distance $f$ behind the FZP, whereas in the second case the source is imaged one-to-one at $z=2 f$. In addition, the imaging properties of zone plate FZP-3 in Table I have been analyzed for a wide range of source distances.

In each wavefield calculation, the number of zones $N$ and the focal length $f$ of the FZP serve as input parameters. From Eq. (1) one obtains the corresponding aperture $D \equiv\left(x_{N}\right.$ $\left.+x_{N-1}\right)$ and outermost zone width $\Delta x_{N}$, see Table I.

For both geometries in Fig. 2, which we label as $f$ and $2 f$, we compare the calculated full widths at half maximum (FWHM) of the images, $w_{f}$ and $w_{2 f}$, with the values expected on the basis of kinematic diffraction from the FZP aperture. Diffraction from a linear aperture causes the image of a point source to broaden according to a $(\operatorname{sinc})^{2}$ intensity distribution. ${ }^{22}$ For an image distance $z$ the FWHM of the central peak of such a distribution is given by

$$
w=0.89 \frac{\lambda z}{D},
$$

with $D / q$ being twice the numerical aperture NA. If the FZP is illuminated by a plane wave, $z=f$. Using Eq. (1) and ne- 
TABLE I. Parameters of the Fresnel zone plates. They have a thickness $h=1.58 \times 10^{4} \mathrm{~nm}$ and $\delta_{\mathrm{Si}}$ $=3.169 \times 10^{-6}$. The wavelength equals $\lambda=0.10 \mathrm{~nm}$. For an explanation of the symbols, see the text.

\begin{tabular}{|c|c|c|c|c|c|c|c|c|c|c|}
\hline \multirow[b]{2}{*}{$\begin{array}{l}\text { Zone } \\
\text { plate }\end{array}$} & \multirow[b]{2}{*}{$N$} & \multirow[b]{2}{*}{$\begin{array}{c}f \\
(\mathrm{~nm})\end{array}$} & \multirow[b]{2}{*}{$\begin{array}{c}D \\
(\mathrm{~nm})\end{array}$} & \multirow[b]{2}{*}{$\begin{array}{l}\Delta x_{N} \\
(\mathrm{~nm})\end{array}$} & \multicolumn{2}{|c|}{$w_{f}(\mathrm{~nm})$} & \multicolumn{2}{|c|}{$w_{2 f}(\mathrm{~nm})$} & \multirow[b]{2}{*}{$\eta_{f}^{\mathrm{a}}$} & \multirow[b]{2}{*}{$\eta_{2 f}^{\mathrm{a}}$} \\
\hline & & & & & $\begin{array}{l}\text { Eq. } \\
(18)\end{array}$ & $\begin{array}{l}\text { This } \\
\text { work }\end{array}$ & $\begin{array}{l}\text { Eq. } \\
\text { (19) }\end{array}$ & $\begin{array}{l}\text { This } \\
\text { work }\end{array}$ & & \\
\hline FZP-1 & 50 & $\begin{array}{r}1.96 \\
\times 10^{7}\end{array}$ & $\begin{array}{c}1.96 \\
\times 10^{4}\end{array}$ & 100 & 89 & 87 & 179 & 145 & 0.403 & 0.398 \\
\hline FZP-2 & 50 & $\begin{array}{c}1.78 \\
\times 10^{4}\end{array}$ & $\begin{array}{c}5.91 \\
\times 10^{2}\end{array}$ & 3.0 & 2.7 & 9.4 & 5.4 & 6.3 & 0.108 & 0.312 \\
\hline FZP-3 & 600 & $\begin{array}{c}5.00 \\
\times 10^{4}\end{array}$ & $\begin{array}{c}3.46 \\
\times 10^{3}\end{array}$ & 1.5 & 1.3 & 10.1 & 2.7 & 3.1 & 0.029 & 0.314 \\
\hline
\end{tabular}

${ }^{\mathrm{a}}$ Reference 20.

glecting its second term, we can approximate the focal distance by $f \simeq D \Delta x_{N} / \lambda$. Therefore we can rewrite Eq. (17) as

$$
w_{f} \simeq 0.89 \cdot \Delta x_{N} \quad(-\infty \rightarrow f) .
$$

For the one-to-one imaging geometry we expect a FWHM of twice this value,

$$
w_{2 f} \simeq 1.78 \cdot \Delta x_{N} \quad(-2 f \rightarrow 2 f) .
$$

The kinematic-diffraction values of $w_{f}$ and $w_{2 f}$, obtained from Eqs. (18) and (19), are listed in Table I. These values serve as a benchmark in our comparisons with our field propagation results; image sizes can in principle only be larger than these values.

All calculations have been carried out for an X-ray wavelength $\lambda=0.10 \mathrm{~nm}$ and a value of $\delta_{\mathrm{Si}}=3.169 \times 10^{-6}$, which corresponds to the decrement of the real part of the refraction index of silicon at the used wavelength, and a height of $h$ $=1.58 \times 10^{4} \mathrm{~nm}$, corresponding to perfectly $\pi$ phase shifting zones. We note that FZP-2 with $\Delta x_{N}=3.0 \mathrm{~nm}$ has its focal

a

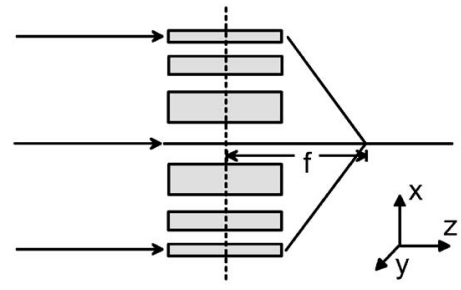

b

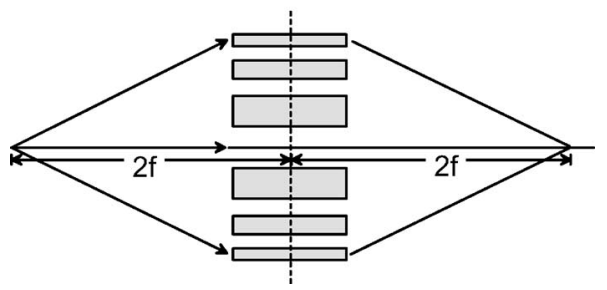

FIG. 2. Use of a linear Fresnel zone plate (FZP) in two different imaging geometries: (a) illumination by a plane wave, resulting in a line focus at a distance $f$ behind the center of the FZP, (b) illumination by cylindrical wave emerging for a line source, located at a distance $2 f$ in front of the FZP and imaged at a distance $2 f$ behind the FZP (one-to-one imaging). point located only $1.0 \times 10^{4} \mathrm{~nm}$ behind its exit, which appears to be unpractical. However, we want to explore fundamental limits rather than practical limits in the focusing properties of FZPs. $^{23}$

The wavefields are numerically propagated using the $\mathrm{C}$ programming language together with standard libraries as provided by Numerical Recipes (NR). ${ }^{24}$ The eigenfunctions and eigenvalues of Eq. (8) are found by diagonalization of the matrix $\mathrm{H}$ in Eqs. (B5) and (B6) by use of the Jacobi algorithm (jacobi) in NR. Wave functions are expressed as a linear combination of free standing-wave solutions using the sine Fourier transform algorithm (sinft) of NR. The number $M$ of independent standing-wave solutions over the interval $a$ in Fig. 1 must be chosen sufficiently large that wavefield variations within the outer zone region are properly described. We have chosen $M=4001$ and $a=2 D$, unless we specify otherwise. For further details we refer to the Appendix. The calculations have been performed on a standard personal computer (PC) equipped with a $3.00 \mathrm{GHz}$ Intel(R) Pentium(R) 4 CPU, 4 GB memory, and a Linux operation system. A typical calculation time for a result as shown, e.g., in Fig. 3 is 0.5 h.

\section{A. Illumination by plane wave}

First we consider the incidence of a plane wave on FZP-1, which has $N=50$ zones and a relatively large outermost zone width $\Delta x_{N}=100 \mathrm{~nm}$. Figure 3(a) shows the evolution of the field amplitude within the volume of the FZP for both the inner and outer zone regions. The large, innermost zones behave very conventionally, with a fairly uniform ( $x$ independent) exit amplitude and a $\pi$-phase shift for the odd zones. As can be seen for the outermost zones, however, refraction from the zone boundary gives rise to interference oscillations in the electric field amplitude as the field propagates through a zone. In the zone cavities the interferences are identical to those occurring between different guided modes within a planar waveguide. ${ }^{25,26}$ In our case, different modes within the cavities are simultaneously excited when the plane wave enters the FZP structure. The field amplitude of the summed modes tends to concentrate in the cavity areas, with each cavity acting as an independent waveguide. The phase and amplitude profile of the wave front exiting the FZP give rise to an image at $z=1.96 \times 10^{7} \mathrm{~nm}[$ Fig. 3(b)]. 

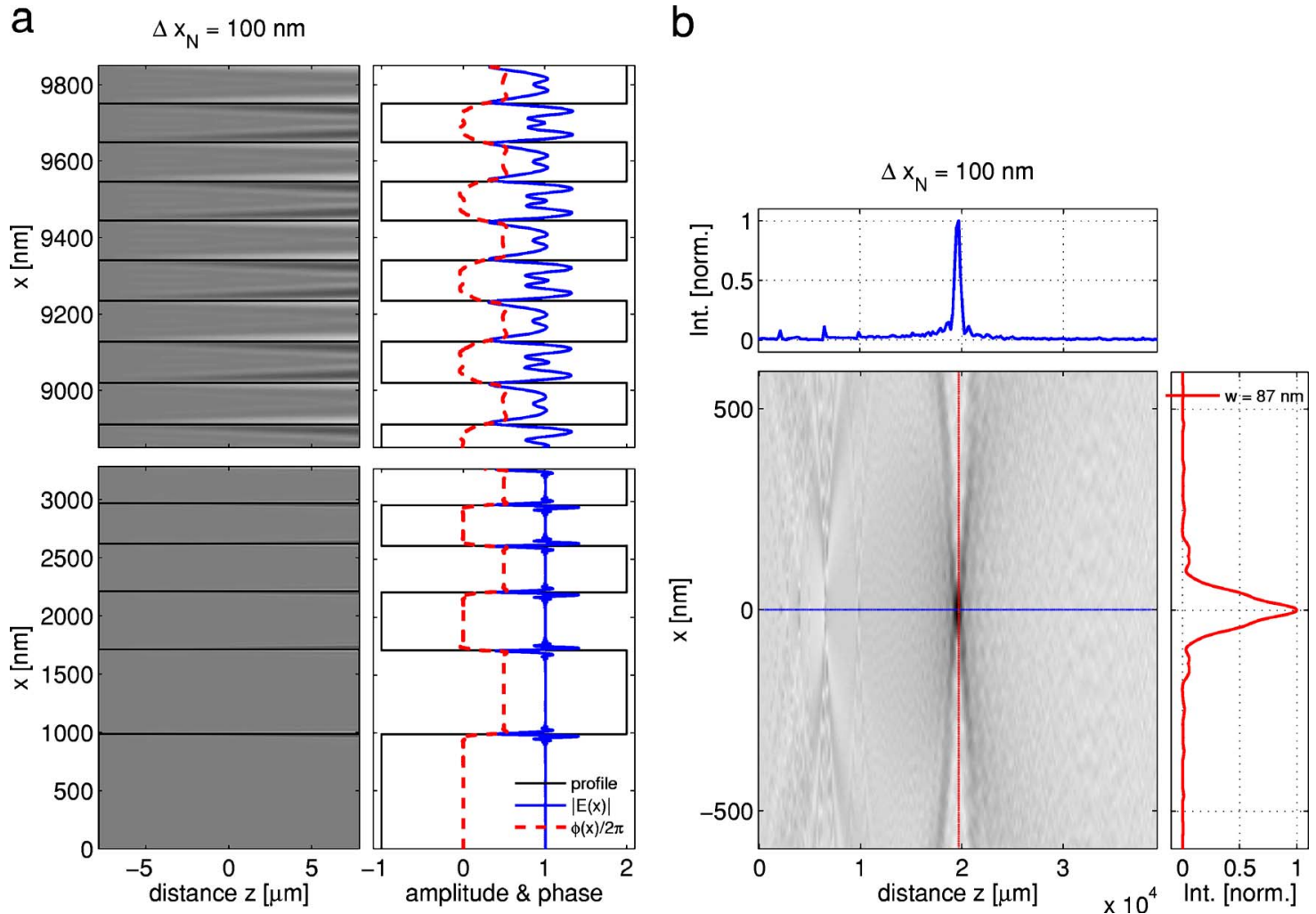

FIG. 3. (Color online) Illumination of FZP-1 with large outermost zone width $\left(\Delta x_{N}=100 \mathrm{~nm}\right)$ by a plane wave. (a) Linear contour plots of the amplitude of the calculated internal wavefield distribution $\Psi(x, z)$ and plots of the amplitude and phase of the exiting wavefield $\Psi$ ex $(x)$. The upper panels show the outermost zones, while the lower panels contain the zones near the center of the FZP. (b) Linear contour plots and slices of the calculated intensity distribution $|\Psi(x, z)|^{2}$ behind the zone plate. Darker regions in the gray scale indicate higher amplitude or intensity.

This image distance equals the focal distance of the zone plate to within the calculational error. For the size $w_{f}$ of the image spot along the $x$ direction we find a value of $87 \mathrm{~nm}$ and for the diffraction efficiency we obtain $\eta_{f}=0.403$. These values agree well with the kinematic predictions, see Table I. The results show that for FZPs with a height much smaller than the focal length, i.e., $h / f<10^{-3}$, the focusing properties are predicted well by the conventional kinematic diffraction formulas, notwithstanding the above-mentioned volume scattering effects.

Next we discuss the properties of FZP-2, which has the same number of zones $(N=50)$ but a much smaller outermost zone width $\Delta x_{N}=3.0 \mathrm{~nm}$. In this case, $h / f \simeq 1$, and significant volume scattering effects are expected. The evolution of the field amplitude within the FZP is shown in Fig. 4(a). Whereas the field in the inner zones tend to concentrate in the cavity regions, as in the previous case, it exhibits much weaker modulations in the outer zone region. This indicates the disappearance of the higher guided waveguide modes from the cavity regions and a delocalization of the lowest mode as discussed in Ref. 11. The amplitude and phase profile of the exiting wavefield now yield a field distribution in image space that is very different from that generated by the FZP with large outermost zone width [Fig. 4(b)].

The wavefront aberrations yield a smeared focus along the $z$ direction, having a FWHM of $w_{f}=9.4 \mathrm{~nm}$ at its maximum along $z$. This spot size is much larger than the outer- most zone width and in considerable disagreement with kinematic value of $2.7 \mathrm{~nm}$ predicted by Eq. (18). In addition, the diffraction efficiency $\eta_{f}=0.108$ is much smaller than the kinematic value. Further calculations for plane-wave incidence (not shown here), where $\Delta x_{N}$ has been varied in the range of 2-8 nm, yield the same results, i.e., a relatively constant spot size of $9-10 \mathrm{~nm}$ and a low diffraction efficiency. We note that this limit on the spot size agrees with the value found previously for a single waveguide. ${ }^{11}$

\section{B. One-to-one imaging}

If the plane incident wave is replaced by a cylindrical wave from a line source located at $z=-2 f$, a limitation on the smallest achievable spot size is no longer observed. The zone plates with $\Delta x_{N}=100 \mathrm{~nm}$ and $\Delta x_{N}=3.0 \mathrm{~nm}$ are now found to produce very similar field distributions in image space and to image the source to $w_{2 f}$ values of $145 \mathrm{~nm}$ and $6.3 \mathrm{~nm}$, respectively (Figs. 5 and 6). These values are comparable to the kinematic-diffraction values of $179 \mathrm{~nm}$ and $5.4 \mathrm{~nm}$ obtained from Eq. (19). In further calculations (not shown here) we have observed a spot size of less than $2 \mathrm{~nm}$ for zone plates with correspondingly small values of $\Delta x_{N}$ under cylindrical-wave illumination.

The FZPs are found to image the source exactly at $z=2 f$. For this one-to-one imaging geometry, the zones are oriented such that for each portion of the FZP the Bragg condition for 

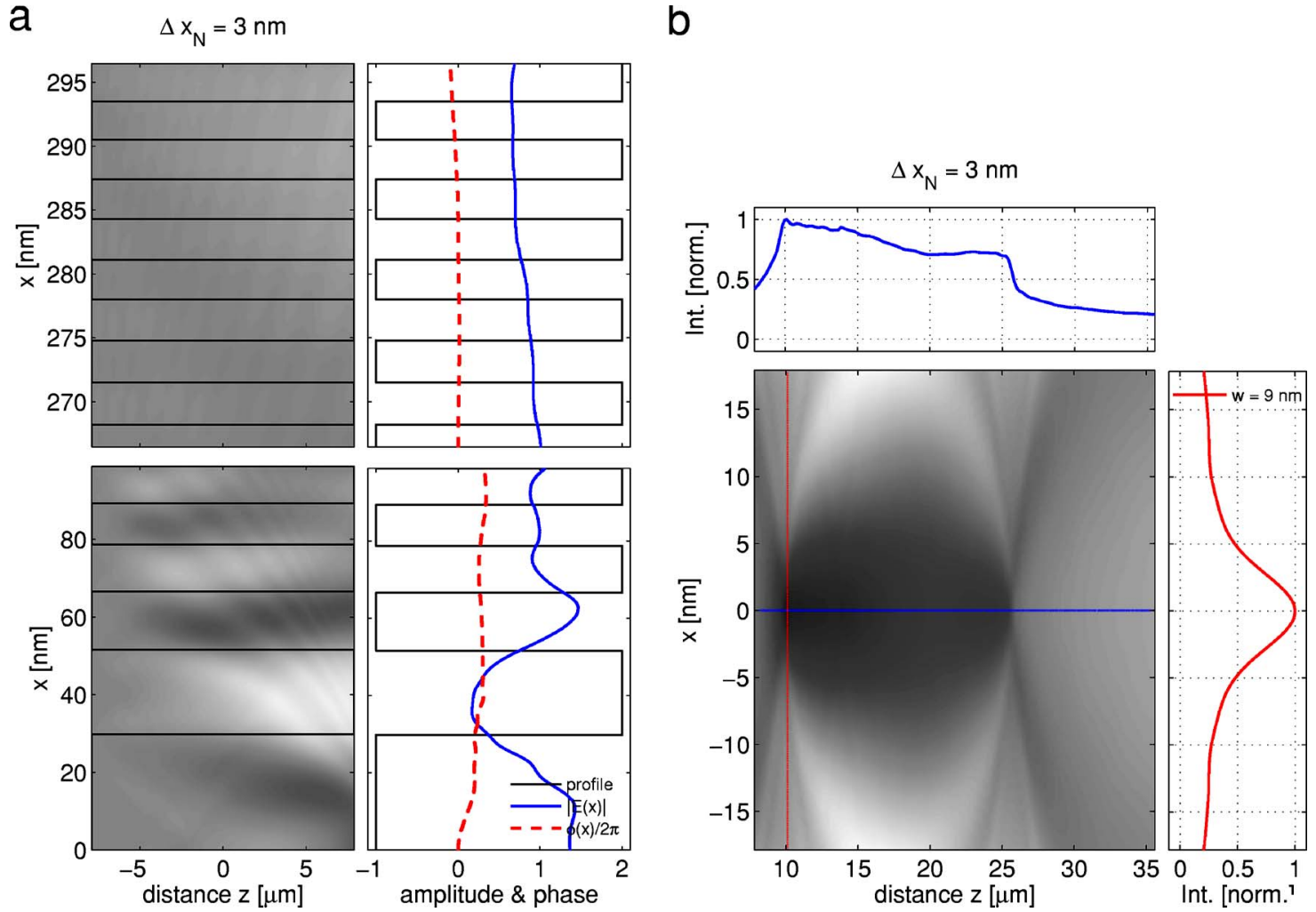

FIG. 4. (Color online) Illumination of FZP-2 with small outermost zone width $\left(\Delta x_{N}=3.0 \mathrm{~nm}\right)$ by a plane wave. (a) Linear contour plots of the amplitude of the calculated internal wavefield distribution $\Psi(x, z)$ and plots of the amplitude and phase of the exiting wavefield $\Psi_{\text {ex }}(x)$. The upper panels show the outermost zones, while the lower panels contain the zones near the center of the FZP. (b) Linear contour plots and slices of the calculated intensity distribution $|\Psi(x, z)|^{2}$ behind the Fresnel zone plate. Darker regions in the gray scale indicate higher amplitude or intensity.

diffraction into the first order is fulfilled. This results not only in an extremely small spot size, but also in a much higher diffraction efficiency $\eta_{2 f}$ in the first order than for the off-Bragg case (see Table I).

Although the FZPs with $\Delta x_{N}=100 \mathrm{~nm}$ and $\Delta x_{N}=3.0 \mathrm{~nm}$ produce similar field distributions in the image space, the field distributions within their volumes are markedly different. This is related to the very different angular apertures of the FZP-1 and FZP-2 in comparison to the critical angle $\theta_{c}$ $=\sqrt{2 \delta_{\mathrm{Si}}}=2.518 \times 10^{-3} \mathrm{rad}$ for total reflection from Si. Since $D /(4 f)$ equals $2.50 \times 10^{-4}$ and $8.85 \times 10^{-3} \mathrm{rad}$, respectively, $\mathrm{x}$ rays are incident at an angle smaller than $\theta_{c}$ with respect to all zone boundaries in the former case and only with respect to a few inner zones in the latter case. Hence, a substantial fraction of the populated waveguide modes in the outer zone region is of the guided type in FZP-1 with $\Delta x_{N}=100 \mathrm{~nm}$ and of the radiative type in FZP-2 with $\Delta x_{N}=3.0 \mathrm{~nm}$.

\section{Variable imaging distance}

With the calculations presented so far, we have explored the two extreme cases of how FZPs can be used as optical elements. Since we have found a considerable difference in the corresponding focusing properties, we will now discuss the focusing limitations of FZPs in cases where the FZP is used as a (de)magnifying optical element, i.e., as a lens. Therefore we investigated the performance of a volume FZP for a line source located at a distance $-\infty<z<-f$. The resulting image size then is a direct measure for the resolution which would be achieved if a FZP is used as, e.g., an objective lens in an $\mathrm{x}$-ray microscope. Figure 7 displays the spot size $w$ (FWHM) in the image plane as a function of the distance of the line source from the FZP center for the zone plate FZP-3 which has an outermost zone width of $\Delta x_{N}$ $=1.5 \mathrm{~nm}$. The image size is found to be smallest when the source is located at a distance $z=-2 f$ from the FZP center, which corresponds to one-to-one imaging and Bragg reflection from the zones into the first order of the FZP. In accordance with what has been found above for FZPs with an outermost zone width considerably smaller than $10 \mathrm{~nm}$, we observe a minimal focus size of $w_{2 f}=3.1 \mathrm{~nm}$, which is approximately equal to the kinematic-diffraction value of $2.7 \mathrm{~nm}$. With increasing source distance, for $z<-2 f$, the image broadens and approaches the limit of the plane-wave case, i.e., $\sim 10 \mathrm{~nm}$. Equally, a broadening of the image size is observed if the source distance is decreased to values of $-2 f<z<-h / 2$.

\section{Fourier spectrum}

It is quite illuminating to look at the Fourier spectrum $\left|\Psi_{\text {ex }}\left(q_{n}\right)\right|$ of the exit wave, given by Eq. (13) and shown in Fig. 8 for a few configurations. In the Bragg geometry, the cutoff frequency $q / 2 \pi$ is given by the inverse of the outer- 

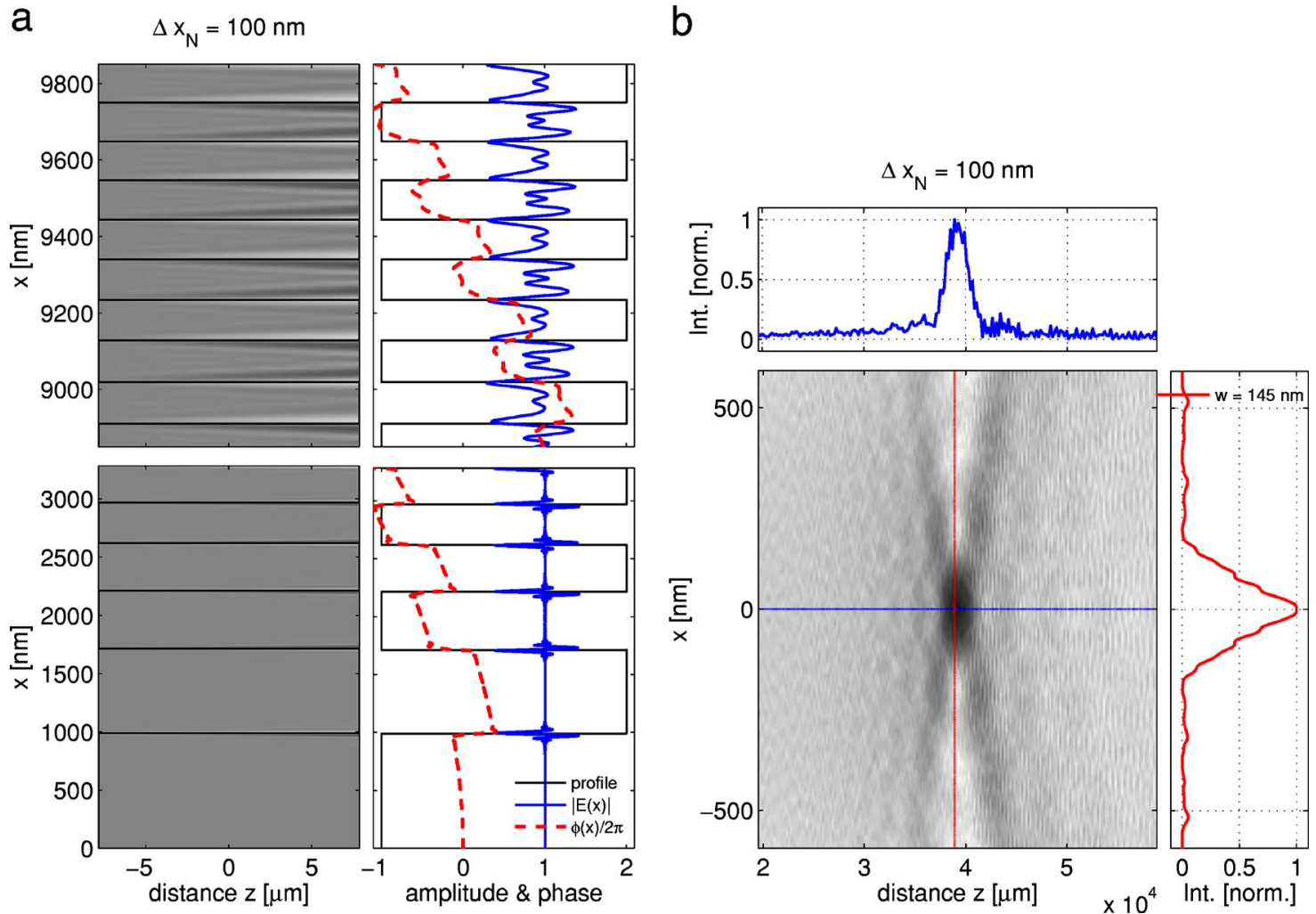

FIG. 5. (Color online) Illumination of FZP-1 with large outermost zone width $\left(\Delta x_{N}=100 \mathrm{~nm}\right)$ by a cylindrical wave from a line source at $z=-2 f$. (a) Linear contour plots of the amplitude of the calculated internal wavefield distribution $\Psi(x, z)$ and plots of the amplitude and phase of the exiting wavefield $\Psi_{\text {ex }}(x)$. The upper panels show the outermost zones, while the lower panels contain the zones near the center of the FZP. Note that the phase profile has been shifted horizontally in the upper right panel to allow for a better representation. (b) Linear contour plots and slices of the calculated intensity distribution $|\Psi(x, z)|^{2}$ behind the Fresnel zone plate. Darker regions in the gray scale indicate higher amplitude or intensity.

most zone width, whereas in the off-Bragg case a much lower cut-off frequency is found for zone plates with small outermost zone width. The size difference between FZP-1 and FZP-2 can be brought out by plotting the Fourier spectra as a function of the normalized wave vector $q_{n} a$. For the Bragg case the curves for FZP-1 and the curve for FZP-2 are found to have identical shapes up over a large interval of $q_{n} a$. In an off-Bragg geometry the highest spatial frequencies are filtered out by FZP-2. This is equivalent to the statement that the local diffraction efficiency decreases in the outer zone region to small values, resulting in a reduced numerical aperture and a correspondingly larger image size.

Rewriting Eq. (13) with the help of (10) as

$$
\left|\Psi_{\text {ex }}\left(q_{n}\right)\right|=\left|\langle\chi_{n} \mid \underbrace{\sum_{m} e^{\mathrm{i} k E_{m} h}\left|\psi_{m}\right\rangle\left\langle\psi_{m}\right|}_{\equiv \mathrm{M}} \Psi_{\mathrm{in}}\rangle\right|
$$

we can see that the Fourier components of the exit wave are the Fourier components of the incoming wave, "scrambled" by the mixing matrix $M$. This matrix is an intrinsic property of the optical device, not a property of the imaging geometry considered.

Written in the basis set of Fourier states, the mixing matrix is diagonally dominant, with off-diagonal components up to a distance $\sim k \sqrt{\delta}$ from the diagonal. In the off-Bragg ge- ometry, in particular for plane wave illumination, the incoming wave profile has very low Fourier components only, and $\left|\Psi_{\text {ex }}\left(q_{n}\right)\right|$ itself cuts off beyond $\sim k \sqrt{\delta}$, giving a broad focus. In the Bragg geometry, the incoming profile already has the Fourier components needed for a much sharper focus, and the mixing matrix essentially rearranges the phases of the Fourier components to construct a converging wave front at the exit.

\section{CONCLUSIONS}

In contrast to X-ray waveguides, Fresnel zone plates possess no fundamental limit to the smallest spot size to which they can focus, provided that they are operated in Bragg geometry for the diffraction order considered. We have demonstrated this for a zone plate with its zones parallel to the optical axis, for which the Bragg geometry in the first diffraction order corresponds with one-to-one imaging with equal distances $(2 f)$ of source and image from the FZP center.

We conclude that for $\mathrm{x}$-ray microscopy applications, where point to point imaging with unequal distances of the source and image plane from the FZP center is desired, a FZP with its zone boundaries lying parallel to the optical axis will be limited in resolution. Although not proven explicitly, 
a
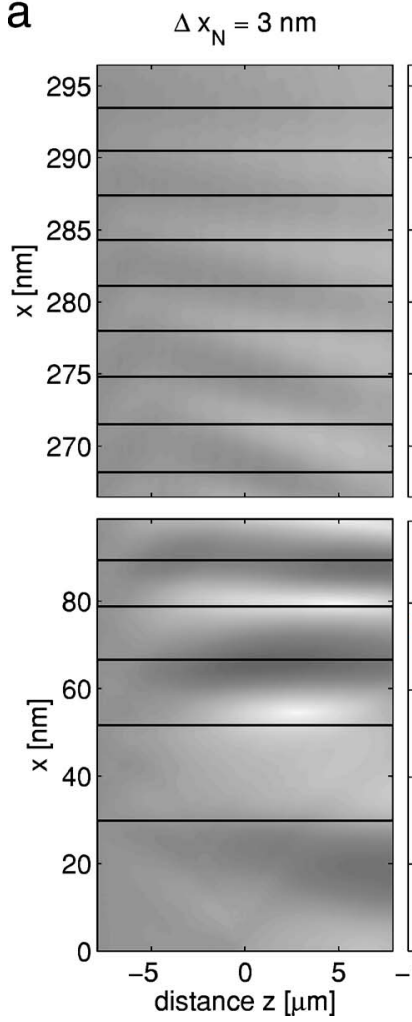
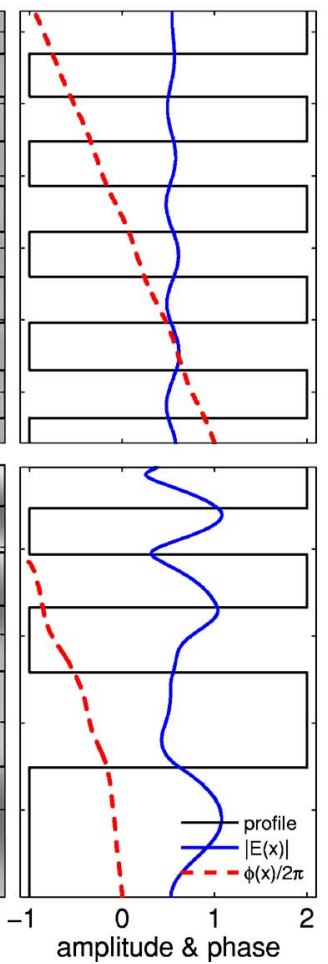

b

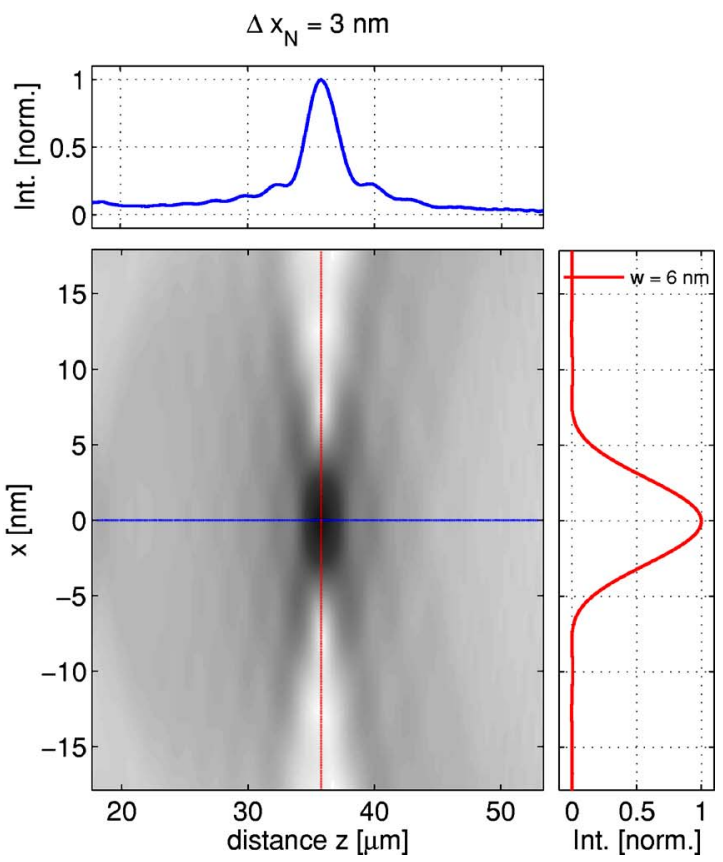

FIG. 6. (Color online) Illumination of FZP-2 with small outermost zone width $\left(\Delta x_{N}=3 \mathrm{~nm}\right)$ by a cylindrical wave from a line source at $z=-2 f$. (a) Linear contour plots of the amplitude of the calculated internal wavefield distribution $\Psi(x, z)$ and plots of the amplitude and phase of the exiting wavefield $\Psi_{\mathrm{ex}}(x)$. The upper panels show the outermost zones, while the lower panels contain the zones near the center of the FZP. Note that the phase profile has been shifted horizontally in the upper right panel to allow for a better representation. (b) Linear contour plots and slices of the calculated intensity distribution $|\Psi(x, z)|^{2}$ behind the Fresnel zone plate. Darker regions in the gray scale indicate higher amplitude or intensity.

the results of our calculation strongly suggest that tilting the zones to the correct Bragg angle, as discussed in Ref. 8, can overcome this fundamental limitation. However, whether it will be possible to image the whole source plane with a resolution corresponding to the resolution of a FZP illuminated by an on-axis point source remains to be shown explicitly.

Note added in proof. After submission of our manuscript, Kang et al. have published a paper that also discusses the focusing limits of Fresnel zone plates [H. C. Kang et al., Phys. Rev. Lett. 96, 127401 (2006)]. Their mathematical approach and the scope of their discussion are different, but they reach broadly similar conclusions regarding the importance of the Bragg condition.

\section{ACKNOWLEDGMENTS}

We are indebted to H.H. Solak for inspiring discussions. C.B. acknowledges the support of the Royal Society.

\section{APPENDIX A: EIGENFUNCTIONS AND EIGENVALUES OF FREE-SPACE HAMILTONIAN $\boldsymbol{H}_{0}$}

The eigenfunctions and eigenvalues of Eq. (11) are readily found. Using Eq. (5) with $V=0$, we have to consider the solutions of

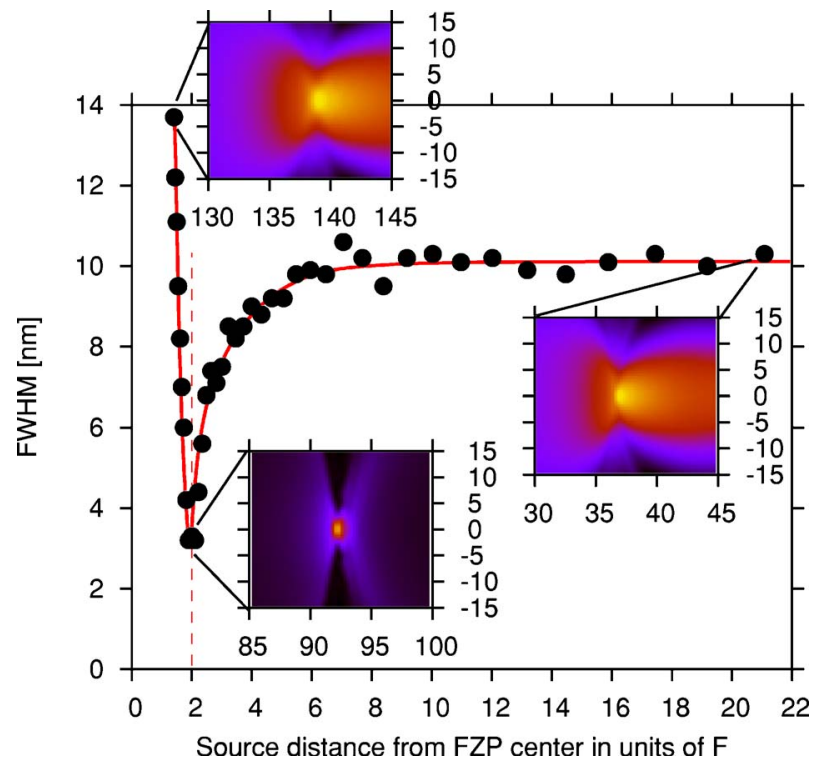

FIG. 7. (Color online) FWHM image size as a function of the distance of the line source from the FZP center in units of $f$, for a FZP with an outermost zone width of $\Delta x_{N}=1.5 \mathrm{~nm} \quad(f=5.0$ $\times 10^{4} \mathrm{~nm}$ ). The following other parameters have been used in the calculations: $N=600, M=6001$, and $a=2.5 D$. 

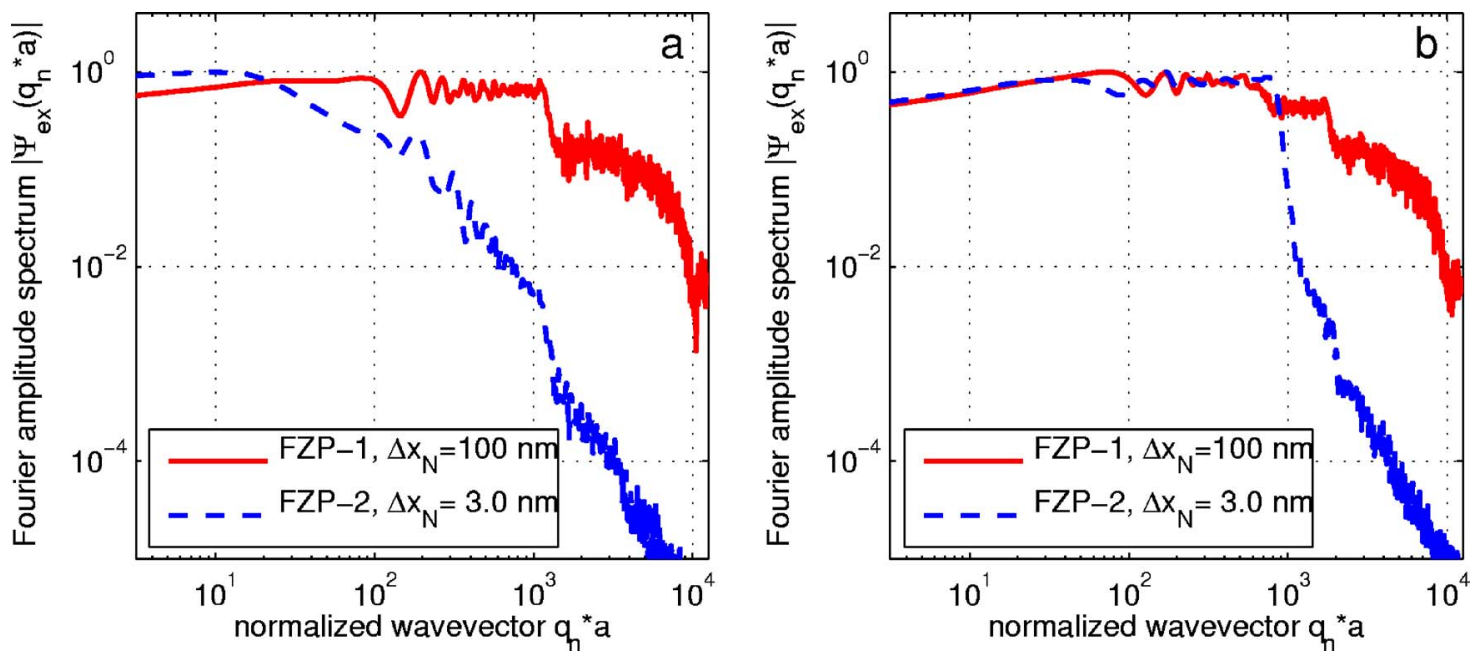

FIG. 8. (Color online) Comparison of the normalized Fourier amplitude spectrum $\left|\Psi_{\text {ex }}\left(q_{n} a\right)\right|$ of FZP-1 and FZP-2 for (a) plane wave illumination and (b) the one-to-one imaging geometry as a function of the normalized wave vector $q_{n} a$.

$$
-\frac{1}{2 k^{2}} \frac{\partial^{2} \chi}{\partial x^{2}}=E^{0} \chi
$$

satisfying the boundary condition that $\chi$ is zero at both ends of the interval $x \in[-a / 2, a / 2]$ (see Fig. 1). Restricting ourselves to even functions around $x=0$, we find the following normalized standing wave solutions:

$$
\chi_{n}(x)=\sqrt{\frac{2}{a}} \cos \left(q_{n} x\right), \quad n=1,2, \ldots
$$

and the corresponding eigenvalues

$$
E_{n}^{0}=\frac{q_{n}^{2}}{2 k^{2}}
$$

with

$$
q_{n} \equiv \frac{(2 n-1) \pi}{a}
$$

the wave vector associated with standing wave $n$. The eigenfunctions $\chi_{n}$ form an orthonormal set,

$$
\left\langle\chi_{n} \mid \chi_{m}\right\rangle=\delta_{n m},
$$

with $\delta_{n m}$ as the Kronecker delta.

\section{APPENDIX B: EIGENFUNCTIONS AND EIGENVALUES OF HAMILTONIAN $H$}

The eigenfunctions and eigenvalues of Eq. (7) are determined by expanding $|\psi\rangle$ in the known eigenfunctions $\left\{\left|\chi_{n}\right\rangle\right\}$ of $H_{0}$,

$$
|\psi\rangle=\sum_{n} c_{n}\left|\chi_{n}\right\rangle
$$

with

$$
c_{n} \equiv\left\langle\chi_{n} \mid \psi\right\rangle
$$

We want to express the Hamiltonian on the basis of the Fourier states $\left\{\left|\chi_{n}\right\rangle\right\}$. The Hamiltonian matrix element of the potential term is

$$
V_{k n} \equiv\left\langle\chi_{k}|V| \chi_{n}\right\rangle=\frac{2}{a} \int_{-a / 2}^{a / 2} \cos q_{k} x \cos q_{n} x \cdot \delta(x) \mathrm{d} x
$$

while the $H_{0}$ matrix elements are of course simply $E_{n}^{0} \delta_{k n}$, since $H_{0}$ is already diagonal in Fourier space.

For the refractive index profile $\delta(x)$ shown in Fig. 1 the integral for $V_{k n}$ can be performed analytically,

$$
\begin{aligned}
V_{k n}= & \delta \cdot \sum_{\nu=1}^{N / 2}\left\{\frac{\sin \left[2(k-n) \pi x_{2 \nu} / a\right]}{(k-n) \pi}-\frac{\sin \left[2(k-n) \pi x_{2 \nu-1} / a\right]}{(k-n) \pi}\right. \\
& +\frac{\sin \left[2(k+n-1) \pi x_{2 \nu} / a\right]}{(k+n-1) \pi} \\
& \left.-\frac{\sin \left[2(k+n-1) \pi x_{2 \nu-1} / a\right]}{(k+n-1) \pi}\right\},
\end{aligned}
$$

where for the diagonal elements $V_{n n}$ the first two terms in the summation reduce to $2 \delta\left(x_{2 \nu}-x_{2 \nu-1}\right) / a$. $N$ in the upper limit of the summation equals the number of zones, which is taken to be an even number.

In matrix notation on the basis of Fourier states we then have

$$
H \mathbf{c}=E \mathbf{c},
$$

with 
$$
\begin{gathered}
H=\left(\begin{array}{cccccc}
E_{1}^{0}+V_{11} & V_{12} & V_{13} & \cdots & \cdots & V_{1 M} \\
V_{21} & E_{2}^{0}+V_{22} & V_{23} & \cdots & \cdots & V_{2 M} \\
V_{31} & V_{32} & \ddots & & & \vdots \\
\vdots & & & & & \vdots \\
V_{M-1,1} & \cdots & & & & V_{M-1, M} \\
V_{M, 1} & V_{M, 2} & \cdots & \cdots & V_{M, M-1} & E_{M}^{0}+V_{M M}
\end{array}\right), \\
\mathbf{c}=\left(\begin{array}{c}
c_{1} \\
c_{2} \\
\vdots \\
\vdots \\
c_{M-1} \\
c_{M}
\end{array}\right) .
\end{gathered}
$$

For computational reasons we can of course only consider finite matrices, cut off beyond the Fourier component with wave vector $q_{M}$. The eigenfunctions and eigenvalues of $H$ are found by diagonalizing the matrix. Because $H$ is real and symmetric, its eigenvalues are real and its eigenfunctions are orthogonal. The dimension $M(\gg N)$ of the matrix has to been chosen such that the maximum spatial frequency of the eigenfunctions is significantly larger than the reciprocal of the outer zone width. Typically, $M$ is in the range of 20005000 , while $N$ can be equal up to a few hundred. In order to avoid that the sine functions in Eq. (B4) would have to be numerically evaluated $\sim 10^{8}$ times, we exploit the fact that $H$ is symmetric and that for a single summation term in Eq. (B4) the first two sine terms are constant along a co-diagonal of $H$ and the latter two terms are constant along an antidiagonal. This speeds up the matrix initialization by typically a factor of $\sim 10^{3}$.
${ }^{1}$ C. G. Schroer, M. Kuhlmann, U. T. Hunger, T. F. Günzler, O. Kurapova, S. Feste, F. Frehse, B. Lengeler, M. Drakopoulos, A. Somogyi, A. S. Simionovici, A. Snigirev, I. Snigireva, C. Schug, and W. H. Schröder, Appl. Phys. Lett. 82, 1485 (2003).

${ }^{2}$ C. G. Schroer, O. Kurapova, J. Patommel, P. Boye, J. Feldkamp, B. Lengeler, M. Burghammer, C. Riekel, L. Vincze, A. van der Hart, and M. Küchler, Appl. Phys. Lett. 87, 124103-1 (2005).

${ }^{3}$ K. Yamauchi, K. Yamamura, H. Mimura, Y. Sano, A. Saito, A. Souvorov, M. Yabashi, K. Tamasaku, T. Ishikawa, and Y. Mori, J. Synchrotron Radiat. 9, 313 (2002).

${ }^{4}$ M. J. Zwanenburg, J. H. H. Bongaerts, J. F. Peters, D. Riese, and J. F. van der Veen, Physica B 283, 285 (2000).

${ }^{5}$ F. Pfeiffer, C. David, M. Burghammer, C. Riekel, and T. Salditt, Science 297, 230 (2002).

${ }^{6}$ C. David, T. Weitkamp, B. Nöhammer, and J. F. van der Veen, Spectrochim. Acta, Part B 59, 1505 (2004).

${ }^{7}$ B. Nöhammer, C. David, M. Burghammer, and C. Riekel, Appl. Phys. Lett. 86, 163804 (2005).

${ }^{8}$ J. Maser, Proc. SPIE 5539, 185 (2004).

${ }^{9}$ W. Chao, B. D. Harteneck, J. A. Liddle, E. H. Anderson, and D. T. Attwood, Nature (London) 435, 1210 (2005).

${ }^{10}$ D. H. Bilderback and R. Huang, AIP Conf. Proc. 705, 1271 (2004).

${ }^{11}$ C. Bergemann, H. Keymeulen, and J. F. van der Veen, Phys. Rev. Lett. 91, 204801 (2003).

${ }^{12}$ C. G. Schroer and B. Lengeler, Phys. Rev. Lett. 94, 054802 (2005).

${ }^{13}$ Y. V. Kopylov, A. V. Popov, and A. V. Vinogradov, Opt. Commun. 118, 619 (1995).

${ }^{14}$ J. Maser and G. Schmahl, Opt. Commun. 89, 355 (1992).
${ }^{15}$ G. Schneider, Appl. Phys. Lett. 71, 2242 (1997).

${ }^{16}$ D. Hambach, G. Schneider, and E. M. Gullikson, Opt. Lett. 26, 1200 (2001).

${ }^{17}$ A. N. Kurokhtin and A. V. Popov, J. Opt. Soc. Am. A 19, 315 (2002).

${ }^{18}$ H. C. Kang, G. B. Stephenson, C. Liu, R. Conley, A. T. Macrander, J. Maser, S. Bajt, and H. N. Chapman, Appl. Phys. Lett. 86, 151109 (2005).

${ }^{19}$ R. Scarmozzino and R. M. Osgood, J. Opt. Soc. Am. B 8, 724 (1991).

${ }^{20}$ In our case the numerical integration in Eq. (14) was carried out over an interval $\left[-6 \times \Delta x_{N}, 6 \times \Delta x_{N}\right]$.

${ }^{21}$ J. Kirz, J. Opt. Soc. Am. 64, 301 (1974).

${ }^{22}$ See, for example, M. Born and E. Wolf, Principles of Optics (Pergamon, Oxford, 1980), Chap. 8.

${ }^{23}$ In practice, FZPs will likely employ phase shifts $\Delta \phi<\pi$ and have corresponding structure heights of $h=\lambda \Delta \phi /(2 \pi \delta)$. With $f$ $\simeq D \Delta x_{N} / \lambda$ and $\delta=\lambda^{2} r_{0} n_{e} /(2 \pi)$, the condition $f \gg h / 2$ becomes equivalent to $\Delta x_{M} \gg \Delta \phi /\left(2 D r_{0} n_{e}\right)$, where $r_{0}$ is the electron radius and $n_{e}$ is the electron density of the FZP material. Note that the condition is independent of $\lambda$.

${ }^{24}$ W. H. Press, B. P. Flannery, S. A. Teukolsky, and W. T. Vetterling, Numerical Recipes in C: The Art of Scientific Computing, 2nd ed., (Cambridge University Press, Cambridge, 1992).

${ }^{25}$ M. J. Zwanenburg, J. F. Peters, J. H. H. Bongaerts, S. A. de Vries, D. L. Abernathy, and J. F. van der Veen, Phys. Rev. Lett. 82, 1696 (1999).

${ }^{26}$ M. J. Zwanenburg, J. F. van der Veen, H. G. Ficke, and H. Neerings, Rev. Sci. Instrum. 71, 1723 (2000). 